\author{
Guillaume Abehsira \\ François Bagate \\ Philippe Estagnasié \\ Pierre Squara
}

\section{Multiple aortic mycotic aneurysms complicating a spondylitis without endocarditis}

Received: 17 January 2013

Accepted: 11 February 2013

Published online: 14 March 2013

(C) Springer-Verlag Berlin Heidelberg and ESICM 2013

G. Abehsira (®) · F. Bagate · P. Estagnasié · P. Squara ICU, Clinique Ambroise Paré, 27 bd Victor Hugo, 92200 Neuilly-sur-Seine, France

e-mail: guillaume.abehsira@hotmail.fr

This angio-MRI shows two aortic mycotic aneurysms in a 75-year-old woman treated for a methicillin-resistant Staphylococcus aureus lumbar spondylitis, following spine surgery. The first aneurysm was located on the aortic isthmus and sized at $35 \mathrm{~mm}$. The second aneurysm was developed at the celiac artery emergence and sized $24 \mathrm{~mm}$ (Fig. 1).

After spondylitis diagnosis, the initial antibiotherapy associated i.v. methicillin and rifampicin. Panton-Valentine leukocidin and endothelial toxin genes were negative. Hepatitis occurred 7 days later, leading to replacement of rifampicin by Levofloxacin. Fever persisted during one week. A transesophageal echocardiography showed no endocarditis, but revealed a thoracic aneurysm. Allograft transplantation was realized after 7 days of a new antibiotherapy combining two previously unused antibiotics with good tissue diffusion (trimethoprim, sulfamethoxazole and clindamycin) plus gentamicin to avoid possible hidden acquired resistance. Direct bacteriological examination of the specimen found the presence of Gram-positive cocci. Histological examination confirmed the mycotic aneurysm. Despite apparent infection control, the distal allograft suture ruptured 1 month after surgery and the patient died.

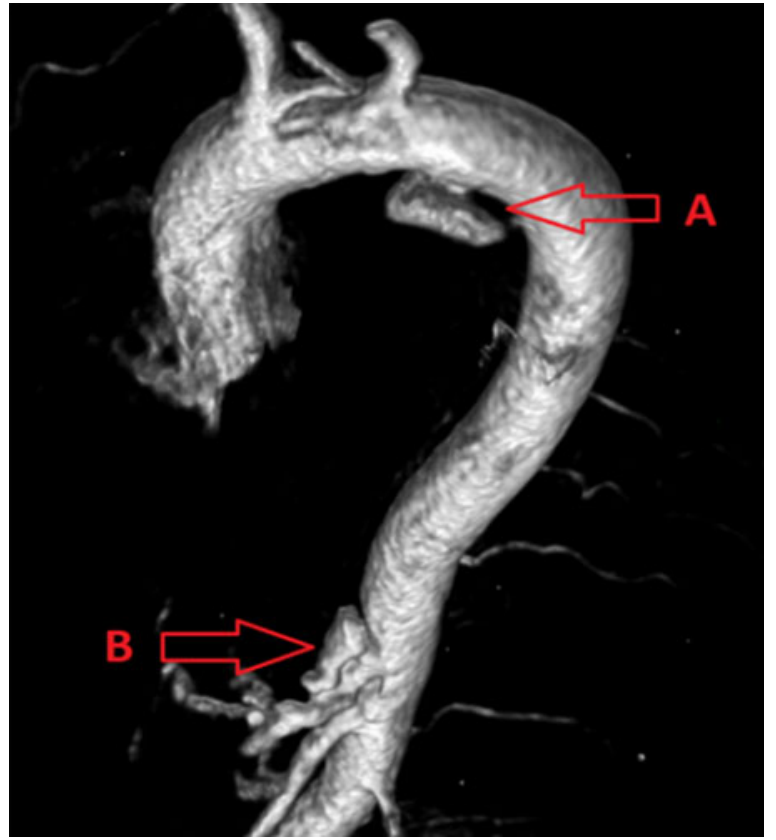

Fig. 1 First aneurysm $(A)$ located immediately after aortic isthmus and sized $35 \mathrm{~mm}$; second aneurysm $(B)$ located at celiac artery emergence and sized $24 \mathrm{~mm}$

This case shows that mycotic aneurysm may occur without endocarditis [1] and may affect aortic walls. The initial antibiotherapy using only one molecule with good tissue diffusion is questionable.

Conflicts of interest Authors state that there is no conflict of interest.

\title{
Reference
}

1. Lehner S, Wittgen C (2005) Infections of the aorta: case report and review of treatment. Vascular 13:252-256 